\title{
Fabrication of Gas Field Ion Emitter by Field Induced Oxygen Etching Method*
}

\author{
Yasushi Sugiura \\ Graduate School of Engineering, University of Mie, 1577 Kurima-Machiya, Tsu 514-8507, Japan \\ Huarong Liu and Tatsuo Iwata \\ Center for Ultimate Technology on nano-Electronics, \\ University of Mie, 1577 Kurima-Machiya, Tsu 514-8507, Japan \\ Shigekazu Nagai and Kazuo Kajiwara \\ Graduate School of Engineering, University of Mie, \\ 1577 Kurima-Machiya, Tsu 514-8507, Japan and \\ Center for Ultimate Technology on nano-Electronics, \\ University of Mie, 1577 Kurima-Machiya, Tsu 514-8507, Japan \\ Koji Asaka and Yahachi Saito \\ Graduate School of Engineering, University of Nagoya, Fro-cho, Chikusa-ku, Nagoya 514-8507, Japan \\ Koichi $\mathrm{Hata}^{\dagger}$ \\ Graduate School of Engineering, University of Mie, \\ 1577 Kurima-Machiya, Tsu 514-8507, Japan and \\ Center for Ultimate Technology on nano-Electronics, \\ University of Mie, 1577 Kurima-Machiya, Tsu 514-8507, Japan \\ (Received 26 July 2011; Accepted 18 August 2011; Published 17 September 2011)
}

\begin{abstract}
Focused ion beam system with a liquid metal ion source has a serious problem which is the pollution of samples due to irradiated ion species. To solve the problem, we have developed a noble gas field ion source which scarcely gives rise to the pollution. In this case, a key issue for the field-ion emitter is the shape of tip-apex to obtain a higher angular current density. Better performance is expected for a shape of tip-apex having a nanoscale-protrusion on the emitter tip with large radius of curvature. For realization of such a shape of tip-apex, field-induced oxygen etching method, which was reported by Onoda et al., was modified and applied to a sharpened tungsten wire which was electrolitically polished. Observation of formed emitter tips by transmission electron microscopy revealed that a nanoprotrusion having a shape of truncated cone with $\sim 5 \mathrm{~nm}$ in diameter and $\sim 4 \mathrm{~nm}$ in height was formed at the center of an emitter tip with $\sim 90 \mathrm{~nm}$ in radius of curvature. [DOI: 10.1380/ejssnt.2011.344]
\end{abstract}

Keywords: Field ion microscopy; Field ionization; Tungsten; Oxygen; Etching

\section{INTRODUCTION}

In recent years, focused ion beam (FIB) systems equipped with gallium liquid metal ion source have been used in wide application fields, e.g., photo-mask repairing for semiconductor devices, micro-fabrication for microelectromechanical systems, sample preparation for transmission electron microscopy (TEM) and so on. However, their systems have a serious problem which is the pollution of samples due to irradiated ion species. Therefore, a noble gas field ion source (GFIS) which scarcely gives rise to the pollution should be urgently developed for FIB systems. In this case, a key issue for GFIS is the shape of tip-apex of field-ion emitter to obtain a higher angular current density $d I / d \Omega$.

G. R. Hanson et al. indicated that an improvement of ion-beam current of GFIS was needed to form a nanostructure on a field ion emitter tip in 1981 [1]. The nanostructure gives rise to a considerable variation of equipotential planes over an emitter tip, which has a focusing

*This paper was presented at 8th International Symposium on Atomic Level Characterizations for New Materials and Devices, Olympic Parktel, Seoul, Republic of Korea, 22-27 May, 2011.

†Corresponding author: hata@elec.mie-u.ac.jp effect on the ion beam. Recently, various methods for preparing emitter tip with a nanoprotrusion which has a triangular pyramidal shape terminated by a single-atom or a trimer have been investigated $[2,3]$. However, if the field strength goes over the threshold of ionization field, the emission of ion beam occurs not only from the tip apex but also from the ridges around the apex. On the other hand, Kobayashi et al. reported the effect of taper angle on ion-beam current in regard to the shape of emitter-shank $[4,5]$. It was described that if the taper angle of emitter-shank becomes smaller, then a larger ion current could be obtained.

Onoda et al. reported field-assisted oxygen etching as a fabrication method for field electron emitter tips terminated by a single-atom [6-8]. In this study, to focus more effectively an ion beam, we modified the field-induced oxygen etching method to optimize the shape of field ion emitter tip with a nanoprotrusion. Subsequently, observation of their formed emitter tips was executed by using TEM.

\section{FORMATION PROCESS OF NANOPROTRUSION BY FIELD-INDUCED OXYGEN ETCHING}

Figures 1(a) to 1(d) show a schematic of the formation process of a nanoprotrusion by field-induced oxygen 


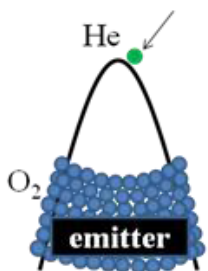

(a)

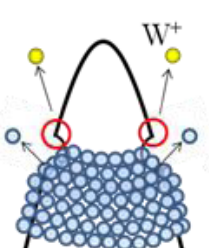

(b)

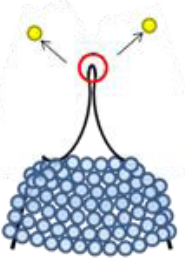

(c)

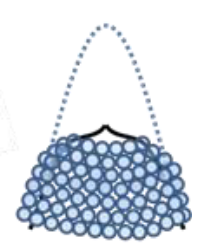

(d)
FIG. 1: Schematic of the formation process (a)-(d) of a nanoprotrusion by a field-induced oxygen etching method.

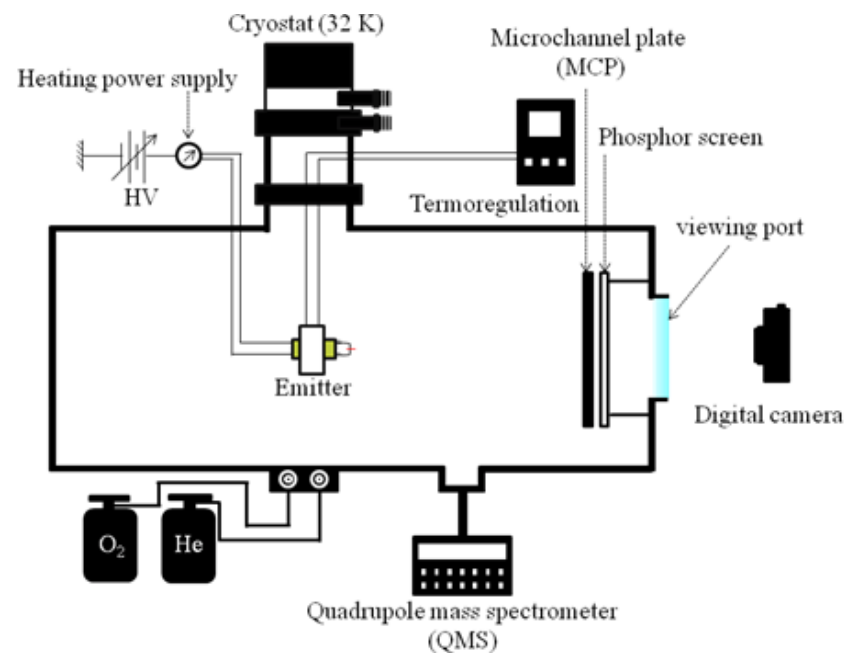

FIG. 2: Schematic of the FIM apparatus.

etching. Under oxygen and helium mixture ambient, high voltage is applied to an emitter tip. Then helium atoms and oxygen molecules are polarized by strong electric field and attracted to the tip-apex. First, helium gas impinges on a tip-apex region, whereas oxygen gas having a lower threshold field for field ionization hardly impinges on a tip-apex under appropriate conditions. Secondly, remaining oxygen gas molecules are adsorbed on an emittershank region with weak field and tungsten is oxidized at the surface layer of this region. Subsequently, tungsten oxide is preferentially field-evaporated because tungsten oxide has a lower threshold field for field evaporation than that of pure tungsten, and then an emitter-shank region is etched off. Thirdly, field evaporation occurs successively from the emitter-shank to tip-apex because of a higher field at an encircled position of sharpened emitter, as shown in Figs. 1(b) and 1(c). Finally, a nanoprotrusion is formed at the center of emitter tip with large radius of curvature.

\section{EXPERIMENTAL PROCEDURES}

First, both $\langle 111\rangle$-oriented single-crystalline tungsten wire with $0.127 \mathrm{~mm}$ in diameter (used for sample \#1) and $\langle 110\rangle$-oriented polycrystalline tungsten wire with $0.15 \mathrm{~mm}$ in diameter (used for sample \#2) were sharpened for field ion emitter tips by the electrolytical-polishing pro-
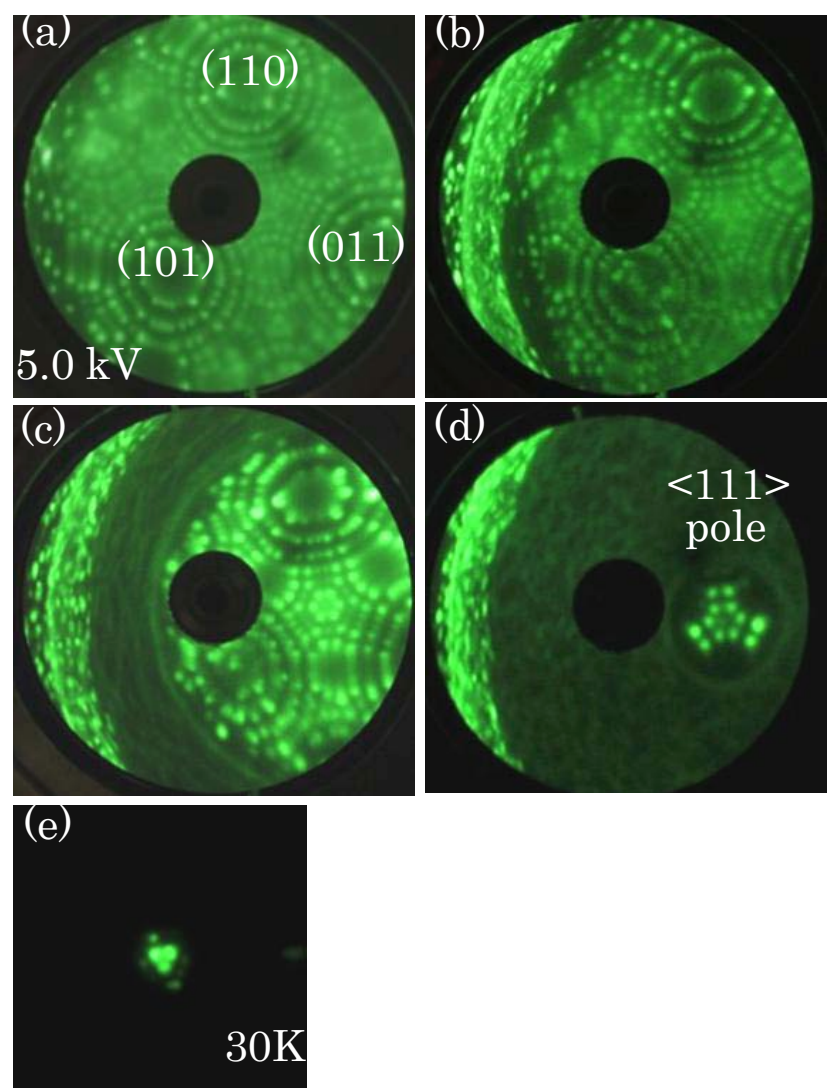

FIG. 3: Sequential changes of FIM images (a)-(d) of the emitter tip during the oxygen etching process (sample tip \#1). (e) FIM image of the emitter tip with trimer obtained by field evaporation process after oxygen etching process.

cess. Then, their emitter tips were installed into a sample chamber of field ion microscope (FIM). Figure 2 shows a schematic of the FIM apparatus used in this study. The sample chamber was evacuated down to ultra-highvacuum of $1.0 \times 10^{-8} \mathrm{~Pa}$. The emitter tip was handled with a manipulator from out of the chamber, and was adjusted to a direction of the phosphor screen. To clean the surface of emitter tips, the flushing process was executed up to $\sim 1000 \mathrm{~K}$, and then their emitter tips were cooled down to $\sim 100 \mathrm{~K}$ by using cryostat.

To observe the surface structure of emitter tip (sample tip \#1), helium as an imaging gas was introduced to a pressure of $\sim 5.0 \times 10^{-3} \mathrm{~Pa}$. High voltage was applied to the tip, and was gradually increased to clean the surface of a tip by field-evaporation process. After the cleaning treatment, oxygen gas was introduced to a pressure of $\sim 2.0 \times 10^{-3} \mathrm{~Pa}$, and then the field-induced oxygen etching process was started. Applied voltage to the tip was kept constant during the whole oxygen etching process. When a nanoprotrusion was formed on the tip-apex, the oxygen etching was stopped by shutting the valve of oxygen gas.

In addition to sample tip \#1 above-mentioned, other emitter tips were prepared for observation of a nanoprotrusion by TEM. The same oxygen etching process was executed for their emitter tips. In this case, the oxygen etching process was stopped on the way to the final stage, as shown in Fig. 1(d). Subsequently, their emitter tips were taken out in air ambient from the FIM cham- 


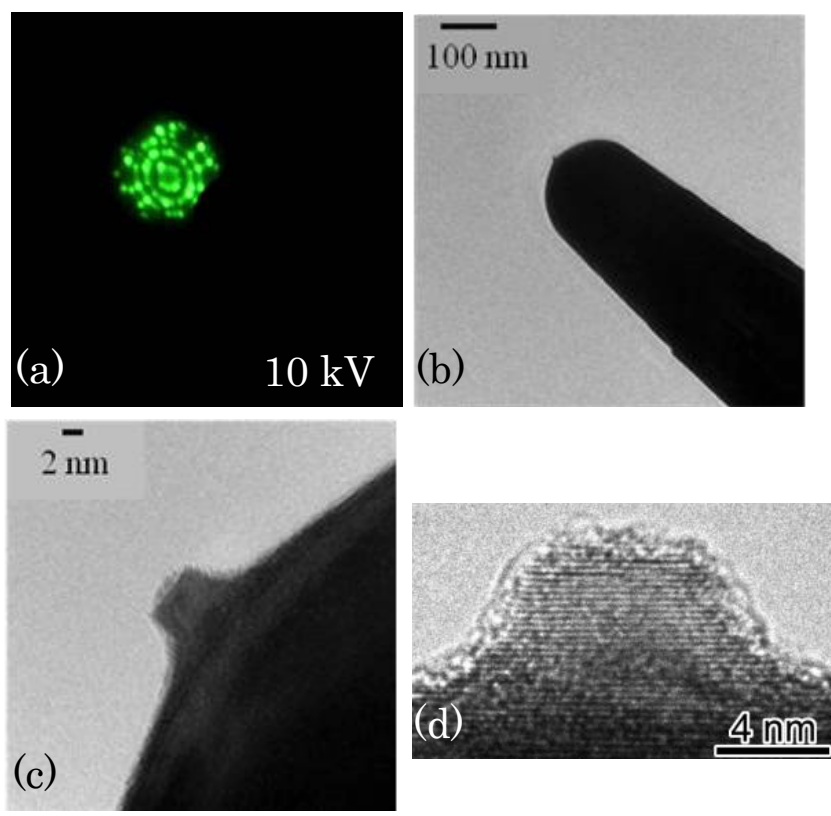

FIG. 4: (a) FIM image of the emitter tip-apex formed by the field-induced oxygen etching (sample tip \#2). (b)-(d) TEM images of the same emitter tip-apex regions as shown in (a).

ber, and were installed into the sample chamber of TEM (JEOL, $200 \mathrm{kV}$ ). Then, observation of a nanostructure of the tip-apex was performed by TEM (sample \#2).

\section{RESULT AND DISCUSSION}

\section{A. Observation of emitter tip by FIM during the formation process}

Figure 3 shows sequential changes of FIM image of the atomic configuration of emitter tip surface (sample tip \#1). Figures 3(a) and 3(b) show FIM images of the clean surface of emitter tip just after the introduction of oxygen gas. Figure 3(a) is a FIM image taken at the tip-apex region, whereas Fig. 3(b) is at the emitter- shank region. A bright zone observed in the left part of Fig. 3(b) was imaged by oxygen ions. Figures $3(\mathrm{c})$ and $3(\mathrm{~d})$ were obtained after 3 hours and 3.5 hours from the start of oxygen etching, respectively. From these figures, it is found that the imaged region at the tip-apex by helium ions gradually became narrow in accordance with the sharpening of an emitter tip. When the FIM image as shown in Fig. 3(d) was reached, oxygen introduction was stopped. Subsequently, etching process by residual and adsorbed oxygen atoms was stopped by quenching the emitter tip to $30 \mathrm{~K}$ at which oxygen freezes. Finally, the tip-apex with a trimer as shown in Fig. 3(e) was obtained by the field evaporation process.

The half angle of beam spread from the obtained trimer was calculated to be $\sim 1.2^{\circ}$. It is found from this value that the beam from emitter tip fabricated by oxygen etching was angular confined significantly.

\section{B. Observation of nanoprotrusion by TEM}

Figure 4(a) shows a FIM image of tip-apex as prepared by the field-induced oxygen etching (sample tip \#2). Then, after taking this emitter tip out from the FIM chamber to air ambient, observation of the fabricated nanoprotrusion was executed by TEM. Figure 4(b) shows a low-magnification TEM image of this emitter tip, and Fig. 4(c) shows a magnified image of tip-apex region. From these images, it was found that a nanoprotrusion, having a shape of truncated cone with $\sim 5 \mathrm{~nm}$ in diameter and $\sim 4 \mathrm{~nm}$ in height, was formed at the center of the emitter tip with $\sim 90 \mathrm{~nm}$ in radius of curvature.

This protrusion was observed by high-resolution TEM, as shown in Fig. 4(d). From this TEM image, the lattice fringe with interplanar spacing of $\sim 0.22 \mathrm{~nm}$ was found in formed protrusion. Since the lattice constant of tungsten is $a=0.3165 \mathrm{~nm}$, the calculated interplanar spacing of tungsten (110) is $0.224 \mathrm{~nm}$. Based on this result, it is found that the formed nanoprotrusion by field-induced oxygen etching was composed of not a contaminant but pure tungsten.

Kalbitzer proposed a shape model of ideal GFIS emitter tip with half angle of beam spread of $\sim 1^{\circ}$, which have a nanoprotrusion centered on an emitter tip with large radius of curvature [9]. It is expected that an emitter tip with such a shape should have a higher $d I / d \Omega$. Therefore, the formation of field ion emitter with an idealized shape of tip-apex was successfully performed by a modified fieldinduced oxygen etching method.

\section{CONCLUSION}

For realization of an idealized shape of field ion emitter for GFIS, a modified field-induced oxygen etching was applied to a sharpened tungsten wire which was electrolitically polished. As a result, by using the modified fieldinduced oxygen etching, the nano-protrusion, having a shape of truncated cone with $\sim 5 \mathrm{~nm}$ in diameter and $\sim 4 \mathrm{~nm}$ in height, was successfully formed at the center of an emitter tip with $\sim 90 \mathrm{~nm}$ in radius of curvature. Also, the beam from the trimer of fabricated emitter tip which have a nano protrusion was angular confined significantly and the half angle of beam spread was $1.2^{\circ}$. In order to evaluate the applicability of the formed emitter to FIB system equipped with GFIS using argon or neon as a feed gas, further investigation is still needed to characterize the angular current density of their field ion emitters in the near future.

\section{Acknowledgments}

This work was supported by the national project, "Development of System and Technology for Advanced Measurement and Analysis", sponsored by the Ministry of Education, Culture, Sports, Science and Technology in Japan. 
[1] G. R. Hanson and B. M. Siegel, Proc. 28th Int. Field Emission Symp. (Oregon Graduate Center, Beaverton, Oregon, 1981), p. 62.

[2] H. S. Kuo, I. S. Hwang, T. Y. Fu, Y. H. Lu, C. Y. Lin, and T. T. Tsong, Appl. Phys. Lett. 92, 063106 (2008).

[3] H. S. Kuo, I. S. Hwang, T. Y. Fu, Y. S. Hwang, Y. H. Lu, C. Y. Lin, J. L. Hou, and T. T. Tsong, Nanotechnology 20, 335701 (2009).

[4] Y. Sugiyama, Y. Kobayashi, Y. Morikawa, K. Kajiwara, and K. Hata, Technical Digest of 22nd Int. Vacuum Nanoelectronics Conf. July 20-24, 2009, Hamamatsu Japan, p. 213.

[5] Y. Kobayashi, Y. Sugiyama, Y. Morikawa, K. Kajiwara, and K. Hata, Technical Digest of 22nd Int. Vacuum Nanoelectronics Conf. July 20-24, 2009, Hamamatsu Japan, p. 215.

[6] F. Rahman, J. Onoda, K. Imaizumi, and S. Mizuno, Surf. Sci. 602, 2128 (2008).

[7] J. Onoda, F. Rahman, and S. Mizuno, e-J. Surf. Sci. Nanotech. 6, 152 (2008).

[8] J. Onoda, S. Mizuno, and H. Ago, Surf. Sci. 604, 1094 (2010).

[9] S. Kalbitzer, Nucl. Instrum. Methods Phys. Res. B 158, 53 (1999). 